\title{
Identifiability of Covariance Kernels in the Gaussian Process Regression Model
}

\author{
Jaehoan Kim${ }^{1}$. Jaeyong Lee ${ }^{2}$ \\ ${ }^{1}$ Department of Mechanical Engineering, Seoul National University \\ ${ }^{2}$ Department of Statistics, Seoul National University
}

\begin{abstract}
Gaussian process regression (GPR) model is a popular nonparametric regression model. In GPR, features of the regression function such as varying degrees of smoothness and periodicities are modeled through combining various covarinace kernels, which are supposed to model certain effects (Bousquet et al., 2011; Gelman et al., 2013). The covariance kernels have unknown parameters which are estimated by the EM-algorithm or Markov Chain Monte Carlo. The estimated parameters are keys to the inference of the features of the regression functions, but identifiability of these parameters has not been investigated. In this paper, we prove identifiability of covariance kernel parameters in two radial basis mixed kernel GPR and radial basis and periodic mixed kernel GPR. We also provide some examples about non-identifiable cases in such mixed kernel GPRs.
\end{abstract}

Keywords: Covariance, Gaussian Process, Identifiability, Kernel, Regression.

\section{Introduction}

Gaussian process regression (GPR) is a widely used nonparametric regression method and can be used in time series analysis. In the GPR modeling, seasonal effects are modeled with periodic covariance kernel and the smoothness of the regression function is with radial basis function (RBF) kernel. Various expansions including Gaussian process with colored noise (Li et al., 1963) were developed.

When observed data are modeled with Gaussian process regression, it is often insufficient to use just one type of covariance kernel. Most of observed features of regression function can be modeled by superposition of several effects such as seasonalities, smoothness, etc. For example, Gelman et al. (2013) modeled weekly, monthly and yearly effects, and short-term and long-term trends by adding covariance kernels of corresponding effects. In Wilson and Adams (2013), several kernels are used for prediction with patterns. In addition to Gaussian process, mixed models are widely in use because of its flexibility (Choi and Lee, 2017; Noh et al., 2018).

\footnotetext{
${ }^{1}$ Undergraduate Student, Department of Mechanical Engineering, Seoul National University, Gwanak, Seoul 08826, Korea. E-mail: k1mjh6561@snu.ac.kr

2 Professor, Department of Statistics, Seoul National University, Gwanak, Seoul 08826, Korea. E-mail: jylc@snu.ac.kr
} 
With the data and mixed model assumption, one can conclude the leverage of each effect with several algorithms mentioned below. For example, in Gelman et al. (2013), daily birthrate from 1969 to 1988 is analyzed with mixed GPR model, including weekend effect, seasonal trend, etc. The effect of each term is provided with graphs. Although this result is obtained under reasonable assumptions and algorithms, the uniqueness of such conclusion is assumed without proof. Here, the identifiability of mixed model becomes crucial. With the assurance of identifiability, at last, one can obtain the credibility of the analysis.

In this paper, we prove the identifiability of RBF-periodic mixed kernel GPR model and 2-RBF mixed kernel GPR model under certain conditions of observed data. The RBF and periodic kernels in the GPR model are used to model trends and periodicities in the data, respectively. The RBF-periodic mixed kernel GPR model is for the data with a general trend and a periodicity. The 2-RBF mixed kernel GPR model is used to model the long-term and short-term trends separately. We give numerical examples of unidentifiable cases with several number of observations.

Started from Teicher (1963), previous identifiability problems are mostly defined and dealt in functional sense (Yakowitz and Spragins, 1968; Kostantinos, 2000; Atienza et al., 2006; Barndorff-Nielsen, 1965), which emphasize analytic aspect.

Practically, as in Bousquet et al. (2011) and Gelman et al. (2013), mixed kernel GPR model is widely in use. The methodologies of the estimation of parameters in mixture models include maximizing the posterior probability distribution function (Gelman et al., 2013; Daemi et al., 2019; Bousquet et al., 2011), Laplace approximation (Bousquet et al., 2011), kernel smoothing (Huang et al., 2014), and the usage of specific form of prior probability density function to improve practical identifiability (Gelman et al., 2013).

In addition to these strategies, this paper provide a firm theoretical basis to justify such method's validity in practical uses. Although the results are limited to mixtures of two kernels, the methodology used in this paper can offer the basis of further research.

This paper is organized as follows. In Section 2, we describe the identifiability problem of RBF-periodic and 2-RBF mixed kernel Gaussian process regression models and our results regarding the problem. In Section 3, we present theoretical proofs about two models, respectively. The numerical cases of non-identifiable model in RBF-periodic, and 2-RBF mixed kernel Gaussian process regression models are given in Section 4. Concluding remarks are given in Section 5 .

\section{Identifiability of Mixed kernel Gaussian Process Regression Model}

\subsection{Notation}

Suppose we observe $n \in \mathbb{N}$ samples with $d$-dimensional covariate and 1-dimensional response $\left(x_{1}, y_{1}\right), \ldots,\left(x_{n}, y_{n}\right)$, where $\mathbb{N}$ is the set of natural numbers. The observations follow Gaussian process regression (GPR) model:

$$
y_{i}=f\left(x_{i}\right)+\epsilon_{i}, \epsilon_{i} \stackrel{i i d}{\sim} N\left(0, \sigma_{0}^{2}\right), i=1, \cdots, n,
$$

where $\sigma_{0}^{2}>0$ is the observational variance, $f(x)$ follows Gaussian process with mean function $\mu(x)$ and covariance kernel $K\left(x, x^{\prime}\right)$, and we denote $f(x) \sim G P(\mu, K)$. Note that under the 
GPR model assumption

$$
\left(f\left(x_{1}\right), \cdots, f\left(x_{n}\right)\right)^{T} \sim N\left(\left(\mu\left(x_{1}\right), \mu\left(x_{2}\right), \ldots, \mu\left(x_{n}\right)\right)^{T}, K\left(x_{1}, x_{2}, \cdots, x_{n}\right)\right),
$$

where $K\left(x_{1}, \cdots, x_{n}\right)=\left[K\left(x_{i}, x_{j}\right)\right]$ represents an $n \times n$ covariance matrix, called a kernel matrix, or Gram matrix. In this paper, we consider two covariance kernel functions, the radial basis function kernel and the periodic kernel.

The RBF kernel is defined as

$$
K_{R B F}\left(x, x^{\prime} ; \theta=(\sigma, l)\right)=\sigma^{2} \times \exp \left(\frac{-\left\|x-x^{\prime}\right\|^{2}}{l^{2}}\right), x, x^{\prime} \in \mathbb{R}^{d}, \sigma, l>0 .
$$

We denote $\theta=(\sigma, l)$, where $\sigma$ is the parameter representing the impact of RBF kernel, and $l$ the smoothness of RBF kernel. The periodic kernel is defined as

$$
K_{\text {period }}\left(x, x^{\prime} ; \eta=(\tau, s)\right)=\tau^{2} \times \exp \left(\frac{-2 \sin ^{2}\left(\pi\left(x-x^{\prime}\right) / p\right)}{s^{2}}\right), \quad x, x^{\prime} \in \mathbb{R}, \tau, s, p>0,
$$

where $\tau$ denotes the impact of the periodic kernel, $s$ the smoothness and $p$ the period of the model. Note that the periodic kernel is defined on $x \in \mathbb{R}$ while the RBF kernel is on $x \in \mathbb{R}^{d}$. If $f \sim G P\left(0, K_{\text {period }}\right)$ with period $p$ and $\left|x-x^{\prime}\right|=k p$ with an integer $k, \operatorname{corr}\left(f(x), f\left(x^{\prime}\right)\right)=1$. In this paper, we will consider the period $p$ as a known constant.

Consider a mixed kernel GPR model with two covariance kernel functions:

$$
y=\mu(x)+f_{1}(x)+f_{2}(x)+\epsilon,
$$

where $\mu$ is a mean function, $f_{1} \sim G P\left(0, K_{1}\right), f_{2} \sim G P\left(0, K_{2}\right)$ and $\epsilon \sim N\left(0, \sigma_{0}^{2}\right)$. In this case, $f_{1}+f_{2} \sim G P\left(0, K_{1}+K_{2}\right)$. For the simplicity of discussion, we assume $\mu(x)=0, \forall x$ for the rest of the paper.

\subsection{Identifiability of mixed kernel Gaussian process regression model}

The kernel matrices of the RBF kernel and the periodic kernel are written as

$$
\begin{aligned}
G_{R B F}=G_{R B F}(\theta) & =\left[K_{R B F}\left(x_{i}, x_{j} ; \theta=(\sigma, l)\right)\right] \\
G_{\text {period }}=G_{\text {period }}(\eta) & =\left[K_{\text {period }}\left(x_{i}, x_{j} ; \eta=(\tau, s)\right)\right] .
\end{aligned}
$$

Consider a RBF-periodic mixed kernel GPR model.

$$
y=f_{1}(x)+f_{2}(x)+\epsilon
$$

where

$$
f_{1}(x) \sim G P\left(0, G_{R B F}(\theta)\right), \quad f_{2}(x) \sim G P\left(0, G_{\text {period }}(\eta)\right), \quad \epsilon \sim N\left(0, \sigma_{0}^{2}\right) .
$$

Due to the affine property of Gaussian distribution,

$$
f_{1}(x)+f_{2}(x) \sim G P\left(0, G_{R B F}(\theta)+G_{\text {period }}(\eta)\right)
$$

holds. If $\theta$ and $\eta$ can be uniquely determined from $G_{R B F}+G_{\text {period }}$, the model is identifiable. 
Theorem 2.1 (Identifiability of RBF-Periodic mixed kernel GPR model) Let $X=$ $\left\{\left|x_{i}-x_{j}\right|: 1 \leq i, j \leq n\right\}$. For the RBF-Periodic mixed GPR model with period $p$, if there exist $\alpha, \beta \in X$ such that $\frac{\alpha}{p} \in \mathbb{N}$ and $\frac{\beta}{p} \notin \mathbb{N}$, the RBF-periodic mixed kernel GPR model is identifiable.

This theorem means that when

$$
G_{R B F}\left(\theta_{1}\right)+G_{\text {period }}\left(\eta_{1}\right)=G_{R B F}\left(\theta_{2}\right)+G_{\text {period }}\left(\eta_{2}\right)
$$

holds,

$$
\theta_{1}=\theta_{2}, \quad \eta_{1}=\eta_{2}
$$

is satisfied.

Similar to RBF-periodic mixed kenrel GPR model, suppose 2-RBF mixed kernel GPR model using (2.2) with

$$
f_{1}(x) \sim G P\left(0, G_{R B F}\left(\theta_{1}\right)\right), f_{2}(x) \sim G P\left(0, G_{R B F}\left(\theta_{2}\right)\right), \epsilon \sim N\left(0, \sigma_{0}^{2}\right) .
$$

To distinguish two RBF kernel matrices in the model, let $l_{1}<l_{2}$ for $\theta_{1}=\left(\sigma_{1}, l_{1}\right), \theta_{2}=$ $\left(\sigma_{2}, l_{2}\right)$.

Theorem 2.2 (Identifiability of 2-RBF mixed model) Let $X=\left\{\left\|x_{i}-x_{j}\right\|: 1 \leq i, j \leq\right.$ $n\}$. If $|X| \geq 4$, the 2 -RBF mixed kernel GPR model is identifiable, where $|X|$ is the cardinality of the set $X$.

This theorem means that for $X$ defined above, if $|X| \geq 4$,

$$
G_{R B F}\left(\theta_{11}\right)+G_{R B F}\left(\theta_{12}\right)=G_{R B F}\left(\theta_{21}\right)+G_{R B F}\left(\theta_{22}\right)
$$

and $l_{11}<l_{12}$ and $l_{21}<l_{22}$,

$$
\theta_{11}=\theta_{12}, \quad \theta_{21}=\theta_{22}
$$

is satisfied. The proof of Theorem 1 and 2 are given in the next section.

\section{Proofs}

\subsection{Proof of Theorem 2.1}

Suppose $X$ satisfies condition mentioned in 2.1. Suppose that $G_{R B F}$ is kernel matrix of RBF kernel and $G_{\text {period }}$ of periodic kernel, with period $p$. In this model, let $\theta_{i}=\left(\sigma_{i}, l_{i}\right)$ and $\eta_{i}=\left(\tau_{i}, s_{i}\right)(i=1,2)$.

Suppose

$$
G_{R B F}\left(\theta_{1}\right)+G_{\text {period }}\left(\eta_{1}\right)=G_{R B F}\left(\theta_{2}\right)+G_{\text {period }}\left(\eta_{2}\right), \quad \forall \theta_{1}, \theta_{2}, \eta_{1}, \eta_{2}
$$

which is equivalent to

$$
K_{R B F}\left(x_{i}, x_{j} ; \theta_{1}\right)+K_{\text {period }}\left(x_{i}, x_{j} ; \eta_{1}\right)=K_{R B F}\left(x_{i}, x_{j} ; \theta_{2}\right)+K_{\text {period }}\left(x_{i}, x_{j} ; \eta_{2}\right),
$$


where $i, j=1, \cdots, n$. Note that $(3.2)$ is equivalent to

$$
\left(\sigma_{1}^{2}, \tau_{1}^{2} \exp \left(-\frac{1}{s_{1}^{2}}\right),-\sigma_{2}^{2},-\tau_{2}^{2} \exp \left(-\frac{1}{s_{2}^{2}}\right)\right)^{T} \cdot v(x)=0, \forall x \in X
$$

where

$$
v(x)=\left(\exp \left(-\frac{x^{2}}{l_{1}^{2}}\right), \exp \left(\frac{\cos (2 \pi x / p)}{s_{1}^{2}}\right), \exp \left(-\frac{x^{2}}{l_{2}^{2}}\right), \exp \left(\frac{\cos (2 \pi x / p)}{s_{2}^{2}}\right)\right)^{T} .
$$

This is because for $x=\left|x_{i}-x_{j}\right|,(3.2)$ is written as

$$
\sigma_{1}^{2} \exp \left(-\frac{x^{2}}{l_{1}^{2}}\right)+\tau_{1}^{2} \exp \left(-\frac{\cos (2 \pi x / p)-1}{s_{1}^{2}}\right)=\sigma_{2}^{2} \exp \left(-\frac{x^{2}}{l_{2}^{2}}\right)+\tau_{2}^{2} \exp \left(-\frac{\cos (2 \pi x / p)-1}{s_{2}^{2}}\right)
$$

which is equivalent to $(3.3)$.

Therefore, when we define $V=\{v(x) \mid x \in X\}$ and $\mathcal{C}(V)$ as a set of linear combinations of $V$, $\operatorname{ker} \mathcal{C}(V)$ includes nonzero vector, and $\operatorname{rank} \mathcal{C}(V) \leq 3$.

We first show that if $l_{1} \neq l_{2}$ and $s_{1} \neq s_{2}, \operatorname{rank} \mathcal{C}(V)=4$, and that if $l_{1}=l_{2}$ and $s_{1} \neq s_{2}$ or $l_{1} \neq l_{2}$ and $s_{1}=s_{2}$, a contradiction occurs. Finally, we finish the proof by showing that if $l_{1}=l_{2}$ and $s_{1}=s_{2}$, then $\sigma_{1}=\sigma_{2}$ and $\tau_{1}=\tau_{2}$, which implies that the model is identifiable. For the proof, we need following lemmas.

Lemma 3.1 If there exists $\alpha, \beta \in X$ such that

$$
\frac{\alpha}{p} \in \mathbb{N}, \frac{\beta}{p} \notin \mathbb{N}
$$

then there exists $m \in \mathbb{N}$ and $q>0$ such that

$$
\frac{q}{p} \notin \mathbb{N},\{0, m p, q, m p+q\} \subset X \text { or }\{0, q, m p-q, m p\} \subset X .
$$

Proof: Without loss of generality, suppose that

$$
X=\left\{x_{1}<x_{2}<\ldots<x_{n}\right\}
$$

and let

$$
a_{i}=x_{i+1}-x_{i}, i=1, \cdots, n-1 \text {. }
$$

Case I. Suppose there exists $a_{i}$ such that $\frac{a_{i}}{p} \in \mathbb{N}$. Let

$$
t=\min \left\{i: 1 \leq i \leq n-1, \frac{a_{i}}{p} \in \mathbb{N}\right\}
$$

Suppose $t \geq 2$. By the definition of $t, \frac{a_{t-1}}{p} \notin \mathbb{N}$. Let $q=a_{t-1}$ and $m=\frac{a_{t}}{p}$. Then $x_{t}-x_{t}=0, x_{t}-x_{t-1}=q, x_{t+1}-x_{t}=a_{t}=m p$, and $x_{t+1}-x_{t-1}=m p+q$. 
Thus, $\{0, m p, q, m p+q\} \subset X$.

Suppose $t=1$. Let

$$
s=\min \left\{i: 1 \leq i \leq n-1, \frac{a_{i}}{p} \notin \mathbb{N}\right\}
$$

Note that the existence of $\mathrm{s}$ follows from (3.4). Since $t=1, s \geq 2$, and thus $\frac{a_{s-1}}{p} \in \mathbb{N}$ and $\frac{a_{s}}{p} \notin \mathbb{N}$. Define $q=a_{s}$, and $m=\frac{a_{s-1}}{p}$. Then,

$$
x_{s}-x_{s}=0, x_{s+1}-x_{s}=a_{s}=q, x_{s}-x_{s-1}=m p \text {, and } x_{s+1}-x_{s-1}=m p+q .
$$

Therefore, $\{0, m p, q, m p+q\} \subset V$.

Case II. Suppose that for all $1 \leq i \leq n, \frac{a_{i}}{p} \notin \mathbb{N}$.

Suppose there exists $(i, j)$ such that $\frac{x_{j}-x_{i}}{p} \in \mathbb{N}$ and $j \leq n-1$. For this $i, j$, define $m=\frac{x_{j}-x_{i}}{p}$ and set $q=a_{j}$. Then

$$
x_{j}-x_{j}=0, x_{j}-x_{i}=m p, x_{j+1}-x_{j}=q, \text { and } x_{j+1}-x_{i}=m p+q,
$$

which satisfies (3.5).

Suppose there does not exist $(i, j)$ such that $\frac{x_{j}-x_{i}}{p} \in \mathbb{N}$ with $j \leq n-1$. This implies that for all $(i, j)$ which satisfies $\frac{x_{j}-x_{i}}{p} \in \mathbb{N}, j=n$. Furthermore, we can see that there should be only one pair of $(\mathrm{i}, \mathrm{j})$. If it is not, for $i_{1}$ and $i_{2}$ such that $\frac{x_{n}-x_{i_{1}}}{p} \in \mathbb{N}$ and $\frac{x_{n}-x_{i_{2}}}{p} \in \mathbb{N}, \frac{\left|x_{i_{1}}-x_{i_{2}}\right|}{p} \in \mathbb{N}$ holds, which contradicts the assumption. Therefore, since $\frac{a_{n-1}}{p} \notin \mathbb{N}, i$ which satisfies $\frac{x_{n}-x_{i}}{p} \in \mathbb{N}$ should satisfy $i \leq n-2$. Define

$$
m=\frac{x_{n}-x_{i}}{p}, \text { and } q=x_{n}-x_{n-1} .
$$

Then

$$
x_{i}-x_{i}=0, x_{n-1}-x_{i}=m p-q, x_{n}-x_{n-1}=q, \text { and } x_{n}-x_{i}=m p,
$$

which means that $\{0, m p-q, q, m p\} \subset X$, where $0<q<m p$. This concludes the proof of Lemma 3.1.

Lemma 3.2 If $k \neq l$ and $a \neq b$, two vectors $\left(e^{k a}, e^{k b}\right)$ and $\left(e^{l a}, e^{l b}\right)$ are linearly independent.

Proof: The conclusion of the lemma is equivalent to $\left|\begin{array}{cc}e^{k a} & e^{l a} \\ e^{k b} & e^{l b}\end{array}\right| \neq 0$ when $k \neq l$ and $a \neq b$. This is trivial because $\left|\begin{array}{ll}e^{k a} & e^{l a} \\ e^{k b} & e^{l b}\end{array}\right|=e^{k a+l b} \times\left(1-e^{(k-l)(a-b)}\right)$. 
Lemma 3.3 For $t, s>0, I(t, s)=\frac{t e^{-t s}}{1-e^{-t s}}$ is monotonely decreasing in $\mathrm{t}$.

Proof: Note $\frac{\partial I}{\partial t}=e^{-t s} \times \frac{1-t s-e^{-t s}}{\left(1-e^{-t s}\right)^{2}}$. When we set $r(x)=1-x-e^{-x}, r^{\prime}(x)=-1+e^{-x}$, which implies that $r$ is monotonely decreasing on $\{x>0\}$. Therefore, for all $t, s>0, \frac{\partial I}{\partial t}=$ $\frac{e^{-t s}}{\left(1-e^{-t s}\right)^{2}} \times r(t s)<0$, and $I(t, s)$ is monotonely decreasing in t.

Lemma 3.4 For $p, l>0$, let $h(x ; p, l)=\frac{\exp \left(-(x+p)^{2} / l^{2}\right)-1}{\exp \left(-p^{2} / l^{2}\right)-1}$. If there exists $x>0$ such that $h\left(x ; p, l_{1}\right)=h\left(x ; p, l_{2}\right)$ for $l_{1}, l_{2}>0$, then $l_{1}=l_{2}$.

Proof: For $x, y, p>0$, define

$$
s(x, y ; p)=\frac{\exp \left(-(x+p)^{2} y^{2}\right)-1}{\exp \left(-p^{2} y^{2}\right)-1} .
$$

Then it is equivalent to show that if there exists $x>0$ such that $s\left(x, 1 / l_{1} ; p\right)=s\left(x, 1 / l_{2} ; p\right)$, $l_{1}=l_{2}$.

$$
\begin{aligned}
\frac{\partial s}{\partial y}= & \frac{2 y\left(1-\exp \left(-p^{2} y^{2}\right)\right)\left(1-\exp \left(-(x+p)^{2} y^{2}\right)\right)}{\left(\exp \left(-p^{2} y^{2}\right)-1\right)^{2}} \times \\
& \left(\frac{(x+p)^{2} \exp \left(-(x+p)^{2} y^{2}\right)}{1-\exp \left(-(x+p)^{2} y^{2}\right)}-\frac{p^{2} \exp \left(-p^{2} y^{2}\right)}{1-\exp \left(-p^{2} y^{2}\right)}\right)
\end{aligned}
$$

When we define

$$
g(x, y ; p)=\frac{2 y\left(1-\exp \left(-p^{2} y^{2}\right)\right)\left(1-\exp \left(-(x+p)^{2} y^{2}\right)\right)}{\left(\exp \left(-p^{2} y^{2}\right)-1\right)^{2}},
$$

the following holds:

$$
\frac{\partial s}{\partial y}=g(x, y ; p)\left(I\left((x+p)^{2}, y^{2}\right)-I\left(p^{2}, y^{2}\right)\right)
$$

When $x, y, p>0, g(x, y ; p)>0$ and $(x+p)^{2}>p^{2}>0$ holds. By Lemma $3.3, \frac{\partial s}{\partial y}<0$. Therefore, if $s\left(x, \frac{1}{l_{1}} ; p\right)=s\left(x, \frac{1}{l_{2}} ; p\right), l_{1}=l_{2}$ holds. This concludes the proof.

Lemma 3.5 Let

$$
v(x)=\left(\exp \left(-\frac{x^{2}}{l_{1}^{2}}\right), \exp \left(-\frac{x^{2}}{l_{2}^{2}}\right), \exp \left(\frac{\cos (2 \pi x / p)}{s_{1}^{2}}\right), \exp \left(\frac{\cos (2 \pi x / p)}{s_{2}^{2}}\right)\right)^{T}
$$

and $V=\{v(x) \mid x \in X\}$. If $l_{1} \neq l_{2}, s_{1} \neq s_{2}$, and

$$
\{0, m p, q, m p+q\} \text { or }\{0, q, m p-q, m p\} \subset X,
$$

then $\operatorname{rank} \mathcal{C}(V)=4$ 
Proof: First, suppose that $(0, m p, q, m p+q) \in X$. Then

$$
\begin{gathered}
v(0)=\left(\begin{array}{c}
1 \\
1 \\
\exp \left(\frac{1}{s_{1}^{2}}\right) \\
\exp \left(\frac{1}{s_{2}^{2}}\right)
\end{array}\right) \quad, \quad v(m p)=\left(\begin{array}{c}
\exp \left(-\frac{m^{2} p^{2}}{l_{1}^{2}}\right) \\
\exp \left(-\frac{-m^{2} p^{2}}{l_{2}^{2}}\right) \\
\exp \left(\frac{1}{s_{1}^{2}}\right) \\
\exp \left(\frac{1}{s_{2}^{2}}\right)
\end{array}\right), \\
v(q)=\left(\begin{array}{c}
\exp \left(-\frac{q^{2}}{l_{1}^{2}}\right) \\
\exp \left(-\frac{q^{2}}{l_{2}^{2}}\right) \\
\exp \left(-\frac{(m p+q)^{2}}{l_{1}^{2}}\right) \\
\exp \left(\frac{\cos (2 \pi q / p)}{s_{1}^{2}}\right) \\
\exp \left(\frac{\cos (2 \pi q / p)}{s_{2}^{2}}\right)
\end{array}\right) \quad v(m p+q)=\left(\begin{array}{c}
l_{2}^{2} \\
\exp \left(\frac{\cos (2 \pi q / p)}{s_{1}^{2}}\right) \\
\exp \left(\frac{\cos (2 \pi q / p)}{s_{2}^{2}}\right)
\end{array}\right) .
\end{gathered}
$$

Define

$$
\begin{gathered}
w_{1}=v(m p)-v(0)=\left(\begin{array}{c}
\exp \left(-\frac{m^{2} p^{2}}{l_{1}^{2}}\right)-1 \\
\exp \left(-\frac{m^{2} p^{2}}{l_{2}^{2}}\right)-1 \\
0 \\
0
\end{array}\right) \\
w_{2}=v(m p+q)-v(q)=\left(\begin{array}{c}
\exp \left(-\frac{(m p+q)^{2}}{l_{1}^{2}}\right)-\exp \left(-\frac{q^{2}}{l_{1}^{2}}\right) \\
\exp \left(-\frac{(m p+q)^{2}}{l_{2}^{2}}\right)-\exp \left(-\frac{q^{2}}{l_{2}^{2}}\right) \\
0 \\
0
\end{array}\right) .
\end{gathered}
$$

Since

$$
\mathcal{C}(\{v(0), v(m p), v(q), v(m p+q)\})=\mathcal{C}\left(\left\{v(0), v(q), w_{1}, w_{2}\right\}\right),
$$

it suffices to show that $\operatorname{rank} \mathcal{C}\left(\left\{v(0), v(q), w_{1}, w_{2}\right\}\right)=4$ or equivalently that $\left\{v(0), v(q), w_{1}, w_{2}\right\}$ is a linearly independent set. Suppose that for $a, b, c, d \in \mathbb{R}, a v(0)+b v(q)+c w_{1}+d w_{2}=0$.

By comparing the $3^{\text {rd }}, 4^{\text {th }}$ components, we can see that

$$
a\left(\begin{array}{l}
\exp \left(\frac{1}{s_{1}^{2}}\right) \\
\exp \left(\frac{1}{s_{1}^{2}}\right)
\end{array}\right)+b\left(\begin{array}{c}
\exp \left(\frac{\cos (2 \pi q / p)}{s_{1}^{2}}\right) \\
\exp \left(\frac{\cos (2 \pi q / p)}{s_{2}^{2}}\right)
\end{array}\right)=\left(\begin{array}{l}
0 \\
0
\end{array}\right)
$$

should be satisfied. By Lemma 3.2, the two 2-dimensional vectors are linearly independent, which implies that $a$ and $b$ are 0 . 
By comparing the $1^{\text {st }}$ and $2^{\text {nd }}$ components, we have

$$
c\left(\begin{array}{c}
\exp \left(-\frac{m^{2} p^{2}}{l_{1}^{2}}\right)-1 \\
\exp \left(-\frac{m^{2} p^{2}}{l_{2}^{2}}\right)-1
\end{array}\right)+d\left(\begin{array}{l}
\exp \left(-\frac{(m p+q)^{2}}{l_{1}^{2}}\right)-\exp \left(-\frac{q^{2}}{l_{1}^{2}}\right) \\
\exp \left(-\frac{(m p+q)^{2}}{l_{2}^{2}}\right)-\exp \left(-\frac{q^{2}}{l_{2}^{2}}\right)
\end{array}\right)=\left(\begin{array}{l}
0 \\
0
\end{array}\right) .
$$

This is equivalent to

$$
B\left(\begin{array}{l}
c \\
d
\end{array}\right)=\left(\begin{array}{l}
0 \\
0
\end{array}\right)
$$

where

$$
B=\left(\begin{array}{ll}
\exp \left(-\frac{m^{2} p^{2}}{l_{1}^{2}}\right)-1, & \exp \left(-\frac{(m p+q)^{2}}{l_{1}^{2}}\right)-\exp \left(-\frac{q^{2}}{l_{1}^{2}}\right) \\
\exp \left(-\frac{m^{2} p^{2}}{l_{2}^{2}}\right)-1, & \exp \left(-\frac{(m p+q)^{2}}{l_{2}^{2}}\right)-\exp \left(-\frac{q^{2}}{l_{2}^{2}}\right)
\end{array}\right) .
$$

Suppose that $(c, d) \neq(0,0)$, which means $\operatorname{det} B=0$. When we define

$$
\begin{gathered}
g(x ; p, l)=\exp \left(-\frac{(x+p)^{2}}{l^{2}}\right)-\exp \left(\frac{x^{2}}{l^{2}}\right), \\
\operatorname{det} B=g\left(0 ; m p, l_{1}\right) g\left(q ; m p, l_{2}\right)-g\left(0 ; m p, l_{2}\right) g\left(q, m p, l_{1}\right)
\end{gathered}
$$

holds. When $x, p>0, g(x ; p, l) \neq 0$ holds. Therefore, $\operatorname{det} B=0$ is equivalent to

$$
\frac{g\left(q ; m p, l_{1}\right)}{g\left(0 ; m p, l_{1}\right)}=\frac{g\left(q ; m p, l_{2}\right)}{g\left(0 ; m p, l_{2}\right)} .
$$

Define

$$
h(x ; p, l)=\frac{\exp \left(-(x+p)^{2} / l^{2}\right)-\exp \left(-x^{2} / l^{2}\right)}{\exp \left(-p^{2} / l^{2}\right)-1} .
$$

Note

$$
h(q ; m p, l)=\frac{g(q ; m p, l)}{g(0 ; m p, l)} .
$$

Consequently, $\operatorname{det} B=0$ is equivalent to

$$
h\left(q ; m p, l_{1}\right)=h\left(q ; m p, l_{2}\right) .
$$

By Lemma 3.4, it is equivalent to $l_{1}=l_{2}$, which contradicts to the fact that $l_{1} \neq l_{2}$. Therefore, the assumption that $\operatorname{det} B \neq 0$ cannot be satisfied, which implies that $(c, d)$ should be $(0,0)$. In conclusion, if

$$
a v(0)+b v(q)+c w_{1}+d w_{2}=0
$$


holds,

$$
(a, b, c, d)=(0,0,0,0) .
$$

Accordingly, $\left\{v(0), v(q), w_{1}, w_{2}\right\}$ is a set of linearly independent vectors, which implies rank $\mathcal{C}(V)=4$.

Now, suppose that $(0, q, m p-q, m p) \in X$. In this case, by the definition of $\mathrm{X}, 0<q<m p$. We can prove the result of the lemma similarly with

$$
B=\left(\begin{array}{ll}
\exp \left(-\frac{m^{2} p^{2}}{l_{1}^{2}}\right)-1, & \exp \left(-\frac{(m p-q)^{2}}{l_{1}^{2}}\right)-\exp \left(-\frac{q^{2}}{l_{1}^{2}}\right) \\
\exp \left(-\frac{m^{2} p^{2}}{l_{2}^{2}}\right)-1, & \exp \left(-\frac{(m p-q)^{2}}{l_{2}^{2}}\right)-\exp \left(-\frac{q^{2}}{l_{2}^{2}}\right)
\end{array}\right) .
$$

Therefore, $\operatorname{det} B=0$ is equivalent to

$$
h\left(-q ; m p, l_{1}\right)=h\left(-q ; m p, l_{2}\right) .
$$

By the definition of $h$,

$$
\frac{1}{h(-x ; p, l)}=h(x ; p-x, l)
$$

holds. Consequently,

$$
h\left(-q ; m p, l_{1}\right)=h\left(-q ; m p, l_{2}\right)
$$

is equivalent to

$$
h\left(q ; m p-q, l_{1}\right)=h\left(q ; m p-q, l_{2}\right) .
$$

Since $0<q<m p$, according to Lemma $3.4, l_{1}=l_{2}$ holds. This contradicts the assumption of Lemma 3.5. Therefore, $\{v(0), v(q), v(m p-q), v(m p)\}$ is a linearly independent set, which means that $\operatorname{rank} \mathcal{C}(\{v(0), v(q), v(m p-q), v(m p)\})=4$.

Therefore, if $G_{R B F}\left(\theta_{1}\right)+G_{\text {period }}\left(\eta_{1}\right)=G_{R B F}\left(\theta_{2}\right)+G_{\text {period }}\left(\eta_{2}\right)$ holds, $l_{1} \neq l_{2}$ and $s_{1} \neq s_{2}$ cannot be satisfied simultaneously. Now we consider two more cases to conclude the proof.

Case I. Suppose $l_{1}=l_{2}$ and $s_{1} \neq s_{2}$. This implies that

$$
\left(\sigma_{1}^{2}-\sigma_{2}^{2}\right) \exp \left(-\frac{x^{2}}{l^{2}}\right)+\tau_{1}^{2} e^{-1 / s_{1}^{2}} \exp \left(\frac{\cos (2 \pi x / p)}{s_{1}^{2}}\right)=\tau_{2}^{2} e^{-1 / s_{2}^{2}} \exp \left(\frac{\cos (2 \pi x / p)}{s_{2}^{2}}\right) .
$$

Define

$$
w(x)=\left(\exp \left(-\frac{x^{2}}{l_{1}^{2}}\right), \exp \left(\frac{\cos (2 \pi x / p)}{s_{1}^{2}}\right), \exp \left(\frac{\cos (2 \pi x / p)}{s_{2}^{2}}\right)\right)^{T}, W=\{w(x) \mid x \in X\} .
$$

To satisfy (3.7), $\operatorname{rank} \mathcal{C}(W) \leq 2$ should be satisfied. However, since

$$
\mathcal{C}(\{w(0), w(m p)\})=\mathcal{C}\left(\left\{(1,0,0)^{T},\left(0, \exp \left(\frac{1}{s_{1}^{2}}\right), \exp \left(\frac{1}{s_{2}^{2}}\right)\right)^{T}\right\}\right)
$$


holds and by Lemma 3.2,

$$
w(q)=\left(\exp \left(-\frac{q^{2}}{l_{1}^{2}}\right), \exp \left(\frac{\cos (2 \pi q / p)}{s_{1}^{2}}\right), \exp \left(\frac{\cos (2 \pi q / p)}{s_{2}^{2}}\right)\right)^{T}
$$

cannot be expressed as a linear combination of $(1,0,0)^{T}$ and $\left(0, \exp \left(1 / s_{1}^{2}\right), \exp \left(1 / s_{2}^{2}\right)\right)^{T}$. Therefore, rank $\mathcal{C}(W)=3$ and $\tau_{1}=\tau_{2}=0$ holds, which contradicts the initial assumption.

Case II. Suppose $l_{1} \neq l_{2}$ and $s_{1}=s_{2}$. Then

$$
\sigma_{1}^{2} \exp \left(-\frac{x^{2}}{l_{1}^{2}}\right)+\left(\tau_{1}^{2}-\tau_{2}^{2}\right) e^{-1 / s_{1}^{2}} \exp \left(\frac{\cos (2 \pi x / p)}{s_{1}^{2}}\right)=\sigma_{2}^{2} \exp \left(-\frac{x^{2}}{l_{2}^{2}}\right)
$$

holds. Define

$$
u(x)=\left(\exp \left(-\frac{x^{2}}{l_{1}^{2}}\right), \exp \left(-\frac{x^{2}}{l_{2}^{2}}\right), \exp \left(\frac{\cos (2 \pi x / p)}{s_{1}^{2}}\right)\right)^{T}, U=\{u(x) \mid x \in X\} .
$$

To satisfy $(3.8)$ with $\sigma_{1} \sigma_{2} \neq 0, \operatorname{rank} \mathcal{C}(U) \leq 2$ should be satisfied. Since

$$
u(0)=\left(1,1, \exp \left(\frac{1}{s_{1}^{2}}\right)\right)^{T}, u(m p)=\left(\exp \left(-\frac{m^{2} p^{2}}{l_{1}^{2}}\right), \exp \left(-\frac{m^{2} p^{2}}{l_{2}^{2}}\right), \exp \left(\frac{1}{s_{1}^{2}}\right)\right)^{T}
$$

are linearly independent vectors by Lemma $3.2, \mathcal{C}(U) \subset \mathcal{C}(\{u(0), u(m p)\})$ should be satisfied to make rank $\mathcal{C}(U) \leq 2$. To satisfy this, $u(m p+q)-u(q)$ or $u(m p-q)-u(q)$ should be included in $\mathcal{C}(\{u(0), u(m p)\})$. For $u(m p+q)-u(q)$, it is equivalent to

$$
u(m p+q)-u(q)=c u(0)+d u(m p)
$$

for some $c$ and $d$. By comparing $3^{r d}$ element of (3.9), $c+d=0$ should hold. Then (3.9) is equivalent to

$$
\left(\begin{array}{l}
\exp \left(-\frac{(m p+q)^{2}}{l_{1}^{2}}\right)-\exp \left(-\frac{q^{2}}{l_{1}^{2}}\right) \\
\exp \left(-\frac{(m p+q)^{2}}{l_{2}^{2}}\right)-\exp \left(-\frac{q^{2}}{l_{2}^{2}}\right)
\end{array}\right)=d\left(\begin{array}{c}
\exp \left(-\frac{m^{2} p^{2}}{l_{1}^{2}}\right)-1 \\
\exp \left(-\frac{m^{2} p^{2}}{l_{2}^{2}}\right)-1
\end{array}\right)
$$

In the proof of Lemma 3.5, we proved that to satisfy

$$
\operatorname{det}\left(\begin{array}{ll}
\exp \left(-\frac{m^{2} p^{2}}{l_{1}^{2}}\right)-1, & \exp \left(-\frac{(m p+q)^{2}}{l_{1}^{2}}\right)-\exp \left(-\frac{q^{2}}{l_{1}^{2}}\right) \\
\exp \left(-\frac{m^{2} p^{2}}{l_{2}^{2}}\right)-1, & \exp \left(-\frac{(m p+q)^{2}}{l_{2}^{2}}\right)-\exp \left(-\frac{q^{2}}{l_{2}^{2}}\right)
\end{array}\right)=0,
$$

$l_{1}=l_{2}$ should hold, which contradicts the assumption. For $u(m p-q)-u(q)$, the proof goes exactly the same way. Therefore, to satisfy

$$
G_{R B F}\left(\theta_{1}\right)+G_{\text {period }}\left(\eta_{1}\right)=G_{R B F}\left(\theta_{2}\right)+G_{\text {period }}\left(\eta_{2}\right)
$$

$l_{1}=l_{2}, s_{1}=s_{2}$ should be held. When this holds, to satisfy

$$
K_{R B F}\left(x_{i}, x_{j} ; \theta_{1}\right)+K_{\text {period }}\left(x_{i}, x_{j} ; \eta_{1}\right)=K_{R B F}\left(x_{i}, x_{j} ; \theta_{2}\right)+K_{\text {period }}\left(x_{i}, x_{j} ; \eta_{2}\right),
$$




$$
\begin{gathered}
\sigma_{1}^{2}-\sigma_{2}^{2}=\tau_{2}^{2}-\tau_{1}^{2} \\
\left(\sigma_{1}^{2}-\sigma_{2}^{2}\right) \exp \left(-\frac{\left(x_{i}-x_{j}\right)^{2}}{l_{1}^{2}}\right)=\left(\tau_{2}^{2}-\tau_{1}^{2}\right) \exp \left(-\frac{2 \sin ^{2}\left(\pi\left(x_{i}-x_{j}\right) / p\right)}{s_{1}^{2}}\right)
\end{gathered}
$$

should hold. (3.10) is obtained by setting $i=j$, and (3.11) by $i>j$. Applying (3.11) for $x_{i}, x_{j}$ which satisfy $x_{i}-x_{j}=m p, \sigma_{1}=\sigma_{2}$ and $\tau_{1}=\tau_{2}$ are obtained.

To sum up, if $G_{R B F}\left(\theta_{1}\right)+G_{\text {period }}\left(\eta_{1}\right)=G_{R B F}\left(\theta_{2}\right)+G_{\text {period }}\left(\eta_{2}\right)$ holds, $\theta_{1}=\theta_{2}$ and $\eta_{1}=\eta_{2}$ should be satisfied, which completes the proof of Theorem 2.1.

\subsection{Proof of Theorem 2.2}

Since here, we consider observation points $\left\{x_{i}: 1 \leq i \leq n\right\}$ as $d$-dimensional vectors, and suppose following condition holds:

$$
X=\left\{\left\|x_{i}-x_{j}\right\|: 1 \leq i, j \leq n\right\},|X| \geq 4
$$

Suppose that $G_{R B F}\left(\theta_{1}\right)$ is the kernel matrix of short-term RBF kernel and $G_{R B F}\left(\theta_{2}\right)$ longterm RBF kernel. In this model, denote $\theta_{i j}=\left(\sigma_{i j}, l_{i j}\right)(i, j=1,2)$, where $j=1,2$ represent parameters of short-term RBF kernel matrix, and long-term RBF kernel matrix each.

According to this notation, $l_{11}<l_{12}, l_{21}<l_{22}$.

$$
G_{R B F}\left(\theta_{11}\right)+G_{R B F}\left(\theta_{12}\right)=G_{R B F}\left(\theta_{21}\right)+G_{R B F}\left(\theta_{22}\right)
$$

means that for all $x_{i}, x_{j}$,

$\sigma_{11}^{2} \exp \left(-\frac{\left(x_{i}-x_{j}\right)^{2}}{l_{11}^{2}}\right)+\sigma_{12}^{2} \exp \left(-\frac{\left(x_{i}-x_{j}\right)^{2}}{l_{12}^{2}}\right)=\sigma_{21}^{2} \exp \left(-\frac{\left(x_{i}-x_{j}\right)^{2}}{l_{21}^{2}}\right)+\sigma_{22}^{2} \exp \left(-\frac{\left(x_{i}-x_{j}\right)^{2}}{l_{22}^{2}}\right)$.

It can be represented in vector form:

$$
\left(\sigma_{11}^{2}, \sigma_{12}^{2},-\sigma_{21}^{2},-\sigma_{22}^{2}\right)\left(\begin{array}{c}
\exp \left(-\frac{\left(x_{i}-x_{j}\right)^{2}}{l_{11}^{2}}\right) \\
\exp \left(-\frac{\left(x_{i}-x_{j}\right)^{2}}{l_{12}^{2}}\right) \\
\exp \left(-\frac{\left(x_{i}-x_{j}\right)^{2}}{l_{21}^{2}}\right) \\
\exp \left(-\frac{\left(x_{i}-x_{j}\right)^{2}}{l_{22}^{2}}\right)
\end{array}\right)=0 .
$$

Therefore, for

$$
v(x)=\left(\begin{array}{c}
\exp \left(-\frac{x^{2}}{l_{11}^{2}}\right) \\
\exp \left(-\frac{x^{2}}{l_{12}^{2}}\right) \\
\exp \left(-\frac{x^{2}}{l_{21}^{2}}\right) \\
\exp \left(-\frac{x^{2}}{l_{22}^{2}}\right)
\end{array}\right), V=\{v(x) \mid x \in X\}
$$


there exists nonzero vector which is orthogonal to all vectors in $V$, which means this vector is orthogonal to all vectors in $\mathcal{C}(V)$. Accordingly, $\operatorname{rank} \mathcal{C}(V) \leq 3$ should be held. We use the same logic as the proof of Theorem 2.1. We first show that if $l_{11}, l_{12}, l_{21}, l_{22}$ are mutually different, rank $\mathcal{C}(V)=4$. Including the case of $l_{11}=l_{22}$ or $l_{21}=l_{22}$, when only one pair of $\left\{l_{11}, l_{12}, l_{21}, l_{22}\right\}$ is duplicated, we show that (3.14) cannot be satisfied. Finally, if $l_{11}=l_{21}$ and $l_{12}=l_{22}$, we show that this implies $\sigma_{11}=\sigma_{21}$ and $\sigma_{12}=\sigma_{22}$, which means that the model is identifiable.

For the proof, we need following lemmas.

Lemma 3.6 Let

$$
B\left(\zeta_{1}, \zeta_{2}, \zeta_{3} ; a, b\right)=\left(\begin{array}{ccc}
\zeta_{1}-1 & \zeta_{1}^{a}-1 & \zeta_{1}^{b}-1 \\
\zeta_{2}-1 & \zeta_{2}^{a}-1 & \zeta_{2}^{b}-1 \\
\zeta_{3}-1 & \zeta_{3}^{a}-1 & \zeta_{3}^{b}-1
\end{array}\right)
$$

If $b>a>1, \zeta_{1}, \zeta_{2}, \zeta_{3}>1$, and $\left(\zeta_{1}-\zeta_{2}\right)\left(\zeta_{2}-\zeta_{3}\right)\left(\zeta_{3}-\zeta_{1}\right) \neq 0$ holds, $\operatorname{det} B \neq 0$.

Proof: Assume $\operatorname{det} B=0$. Then there exists $\left(u_{1}, u_{2}, u_{3}\right) \neq(0,0,0)$ such that

$$
u_{1}\left(\begin{array}{l}
\zeta_{1}-1 \\
\zeta_{2}-1 \\
\zeta_{3}-1
\end{array}\right)+u_{2}\left(\begin{array}{l}
\zeta_{1}^{a}-1 \\
\zeta_{2}^{a}-1 \\
\zeta_{3}^{a}-1
\end{array}\right)+u_{3}\left(\begin{array}{c}
\zeta_{1}^{b}-1 \\
\zeta_{2}^{b}-1 \\
\zeta_{3}^{b}-1
\end{array}\right)=0 .
$$

When we define

$$
h(t)=u_{1}(t-1)+u_{2}\left(t^{a}-1\right)+u_{3}\left(t^{b}-1\right),
$$

$\zeta_{1}, \zeta_{2}, \zeta_{3}$ are mutually different roots of $h(t)=0$. With $h(1)=0, h(t)=0$ has 4 mutually different roots on $\{t \geq 1\}$. By mean value theorem,

$$
h^{\prime}(t)=u_{1}+a u_{2} t^{a-1}+b u_{3} t^{b-1}=0
$$

has at least 3 different roots on $\{t>1\}$. By dividing into cases, we prove this cannot be the case.

Case I. Suppose $u_{1}=0, u_{2}=0 . u_{3} \neq 0$ not to make $\left(u_{1}, u_{2}, u_{3}\right)$ zero vector. However, according to (3.16), $h^{\prime}(t)=b u_{3} t^{b-1}$, which means $h^{\prime}(t) \neq 00$ on $\{t>=1\}$. This also implies that $u_{1}=0$ and $u_{3}=0$ should not be satisfied simultaneously.

Case II. Suppose $u_{1}=0, u_{2} \neq 0$ and $u_{3} \neq 0$. According to (3.16),

$$
h^{\prime}(t)=a u_{2} t^{a-1}\left(1+\frac{b u_{3}}{a u_{2}} t^{b-a}\right)
$$

holds. Define

$$
P_{1}(t)=1+\frac{b u_{3}}{a u_{2}} t^{b-a} .
$$

Then $P_{1}(t)=0$ should have at least 3 roots on $\{t \geq 1\}$. This contradicts the fact that $P_{1}(t)$ is monotone function on $\{t \geq 1\}$, since

$$
P_{1}^{\prime}(t)=\frac{b(b-a) u_{3}}{a u_{2}} t^{b-a-1}
$$


does not change its sign on $\{t \geq 1\}$.

Case III. Suppose $u_{1} \neq 0$. By dividing 3.16 by $u_{1}$,

$$
h^{\prime}(t)=u_{1}\left(1+\frac{a u_{2}}{u_{1}} t^{a-1}+\frac{b u_{3}}{u_{1}} t^{b-1}\right)
$$

holds. Define

$$
P_{2}(t)=1+\frac{a u_{2}}{u_{1}} t^{a-1}+\frac{b u_{3}}{u_{1}} t^{b-1} .
$$

Then $P_{2}(t)=0$ should have at least 3 different roots on $\{t>1\}$. If $u_{2}=u_{3}=0$,

$$
h(t)=u_{1}(t-1)+u_{2}\left(t^{a}-1\right)+u_{3}\left(t^{b}-1\right)=u_{1}(t-1)
$$

cannot have 4 different roots on $\{t \geq 1\}$. Similarly, if $u_{2}=0$ or $u_{3}=0, P_{2}^{\prime}(t)$ cannot be 0 on $\{t>1\}$, which implies that $P_{2}$ is monotone, and cannot have 4 different roots on $\{t \geq 1\}$. Suppose $u_{1} u_{2} u_{3} \neq 0$. Applying mean value theorem again, $P_{2}^{\prime}(t)=0$ should have at least 2 different roots on $\{t>1\}$. Denote the smallest root and the second smallest root as $c_{1}, c_{2}$ respectively. Note

$$
P_{2}^{\prime}(t)=\frac{a(a-1) u_{2}}{u_{1}} t^{a-2}+\frac{b(b-1) u_{3}}{u_{1}} t^{b-2},
$$

which can be written as

$$
P_{2}^{\prime}(t)=\frac{a(a-1) u_{2}}{u_{1}} t^{a-2}\left(1+\frac{b(b-1) u_{3}}{a(a-1) u_{2}} t^{b-a}\right) .
$$

Define

$$
P_{3}(t)=1+\frac{b(b-1) u_{3}}{a(a-1) u_{2}} t^{b-a} .
$$

To satisfy $P_{2}^{\prime}\left(c_{1}\right)=P_{2}^{\prime}\left(c_{2}\right)=0, P_{3}\left(c_{1}\right)=P_{3}\left(c_{2}\right)=0$ should hold. However, $P_{3}(t)$ is a monotone function, which contradicts above. Therefore, the assumption is wrong, which concludes that $\operatorname{det} B \neq 0$.

Lemma 3.7 Suppose that $l_{11}, l_{12}, l_{21}, l_{22}$ are mutually different. When we define

$$
v(x)=\left(\begin{array}{l}
\exp \left(-\frac{x^{2}}{l_{11}^{2}}\right) \\
\exp \left(-\frac{x^{2}}{l_{12}^{2}}\right) \\
\exp \left(-\frac{x^{2}}{l_{21}^{2}}\right) \\
\exp \left(-\frac{x^{2}}{l_{22}^{2}}\right)
\end{array}\right),
$$

and $V=\{v(x) \mid x \in X\}, \operatorname{rank} \mathcal{C}(V)=4$. 
Proof: According to (3.12), there exists

$$
a_{1}, a_{2}, a_{3} \in X \text { such that } a_{3}>a_{2}>a_{1}>0 .
$$

Let

$$
a=\frac{a_{2}}{a_{1}}, b=\frac{a_{3}}{a_{1}}, \alpha=\exp \left(-\frac{a_{1}^{2}}{l_{11}^{2}}\right), \beta=\exp \left(-\frac{a_{1}^{2}}{l_{12}^{2}}\right), \gamma=\exp \left(-\frac{a_{1}^{2}}{l_{21}^{2}}\right), \delta=\exp \left(-\frac{a_{1}^{2}}{l_{22}^{2}}\right) .
$$

Since $l_{11}<l_{12}$ and $l_{21}<l_{22}$, without loss of generality, suppose

$$
\min \left(l_{11}, l_{12}, l_{21}, l_{22}\right)=l_{11}
$$

For

$$
v(0)=\left(\begin{array}{l}
1 \\
1 \\
1 \\
1
\end{array}\right), v\left(a_{1}\right)=\left(\begin{array}{l}
\alpha \\
\beta \\
\gamma \\
\delta
\end{array}\right), v\left(a_{2}\right)=\left(\begin{array}{l}
\alpha^{a} \\
\beta^{a} \\
\gamma^{a} \\
\delta^{a}
\end{array}\right), v\left(a_{3}\right)=\left(\begin{array}{c}
\alpha^{b} \\
\beta^{b} \\
\gamma^{b} \\
\delta^{b}
\end{array}\right),
$$

$\mathcal{C}\left(\left\{v(0), v\left(a_{1}\right), v\left(a_{2}\right), v\left(a_{3}\right)\right\}\right) \subset \mathcal{C}(V)$. Therefore, it is enough to show that $\operatorname{rank} \mathcal{C}\left(\left\{v(0), v\left(a_{1}\right), v\left(a_{2}\right), v\left(a_{3}\right)\right\}\right)=4$.

Define

$$
A=\left(\begin{array}{cccc}
1 & 1 & 1 & 1 \\
\alpha & \beta & \gamma & \delta \\
\alpha^{a} & \beta^{a} & \gamma^{a} & \delta^{a} \\
\alpha^{b} & \beta^{b} & \gamma^{b} & \delta^{b}
\end{array}\right)
$$

It is equivalent to show that $\operatorname{det} A \neq 0$. Define

$$
\zeta_{1}=\frac{\beta}{\alpha}, \zeta_{2}=\frac{\gamma}{\alpha}, \zeta_{3}=\frac{\delta}{\alpha}
$$

Since

$$
\min \left(l_{11}, l_{12}, l_{21}, l_{22}\right)=l_{11}, \min (\alpha, \beta, \gamma, \delta)=\alpha,
$$

$\zeta_{1}, \zeta_{2}, \zeta_{3}>1$ holds. Also,

$$
\operatorname{det} A=\alpha^{1+a+b}\left|\begin{array}{cccc}
1 & 1 & 1 & 1 \\
1 & \zeta_{1} & \zeta_{2} & \zeta_{3} \\
1 & \zeta_{1}^{a} & \zeta_{2}^{a} & \zeta_{3}^{a} \\
1 & \zeta_{1}^{b} & \zeta_{2}^{b} & \zeta_{3}^{b}
\end{array}\right|=\left|\begin{array}{cccc}
1 & 1 & 1 & 1 \\
0 & \zeta_{1}-1 & \zeta_{2}-1 & \zeta_{3}-1 \\
0 & \zeta_{1}^{a}-1 & \zeta_{2}^{a}-1 & \zeta_{3}^{a}-1 \\
0 & \zeta_{1}^{b}-1 & \zeta_{2}^{b}-1 & \zeta_{3}^{b}-1
\end{array}\right|=\operatorname{det} B
$$

holds when we define

$$
B=\left(\begin{array}{lll}
\zeta_{1}-1 & \zeta_{2}-1 & \zeta_{3}-1 \\
\zeta_{1}^{a}-1 & \zeta_{2}^{a}-1 & \zeta_{3}^{a}-1 \\
\zeta_{1}^{b}-1 & \zeta_{2}^{b}-1 & \zeta_{3}^{b}-1
\end{array}\right)
$$

By Lemma 3.6, $\operatorname{det} B \neq 0$, which means that $\operatorname{det} A \neq 0$. Therefore, $\operatorname{rank} \mathcal{C}(V)=4$. 
Consequently, to satisfy $(3.13), l_{11}, l_{12}, l_{21}, l_{22}$ should not be mutually different.

Lemma 3.8 For mutually different real numbers $a, b, c>0$, define

$$
w(x)=\left(\begin{array}{c}
\exp \left(-\frac{x^{2}}{a^{2}}\right) \\
\exp \left(-\frac{x^{2}}{b^{2}}\right) \\
\exp \left(-\frac{x^{2}}{c^{2}}\right)
\end{array}\right) .
$$

Then for mutually different real numbers $x_{1}, x_{2}, x_{3}>0,\left\{w\left(x_{1}\right), w\left(x_{2}\right), w\left(x_{3}\right)\right\}$ is a linearly independent set.

Proof: Without loss of generality, suppose

$$
0<x_{1}<x_{2}<x_{3}, a>b>c .
$$

Define

$$
\alpha=\exp \left(-\frac{x_{1}^{2}}{a^{2}}\right), \beta=\exp \left(-\frac{x_{1}^{2}}{b^{2}}\right), \gamma=\exp \left(-\frac{x_{1}^{2}}{c^{2}}\right), A=\frac{x_{2}^{2}}{x_{1}^{2}}, B=\frac{x_{3}^{2}}{x_{1}^{2}}
$$

Then

$$
\alpha>\beta>\gamma, 1<A<B
$$

holds. Define matrix $\mathrm{C}$ as

$$
C=\left(w\left(x_{1}\right), w\left(x_{2}\right), w\left(x_{3}\right)\right)=\left(\begin{array}{ccc}
\alpha & \alpha^{A} & \alpha^{B} \\
\beta & \beta^{A} & \beta^{B} \\
\gamma & \gamma^{A} & \gamma^{B}
\end{array}\right)
$$

Then it is equivalent to show that $\operatorname{det} C \neq 0$. In addition,

$$
\begin{aligned}
\operatorname{det} C=\alpha \beta \gamma\left|\begin{array}{ccc}
1 & \alpha^{A-1} & \alpha^{B-1} \\
1 & \beta^{A-1} & \beta^{B-1} \\
1 & \gamma^{A-1} & \gamma^{B-1}
\end{array}\right| & =\alpha^{A+B-1} \beta \gamma\left|\begin{array}{ccc}
1 & 1 & 1 \\
1 & \left(\frac{\beta}{\alpha}\right)^{A-1} & \left(\frac{\beta}{\alpha}\right)^{B-1} \\
1 & \left(\frac{y}{\alpha}\right)^{A-1} & \left(\frac{y}{\alpha}\right)^{B-1}
\end{array}\right| \\
& =\alpha^{A+B-1} \beta \gamma\left|\begin{array}{ccc}
1 & 1 & 1 \\
0 & \left(\frac{\beta}{\alpha}\right)^{A-1}-1 & \left(\frac{\beta}{\alpha}\right)^{B-1}-1 \\
0 & \left(\frac{\gamma}{\alpha}\right)^{A-1}-1 & \left(\frac{\gamma}{\alpha}\right)^{B-1}-1
\end{array}\right|
\end{aligned}
$$

holds. According to the following lemma, when we set $x_{1}=\frac{\beta}{\alpha}$ and $x_{2}=\frac{\gamma}{\alpha}, \operatorname{det} C=0$ is equivalent to the fact that $x_{1}=x_{2}$, which contradicts the assumption. This concludes the proof. 
Lemma 3.9 for $0<x_{1}, x_{2}<1$, if $x_{1} \neq x_{2}$ and $1<A<B$,

$$
\left|\begin{array}{ll}
x_{1}^{A-1}-1 & x_{1}^{B-1}-1 \\
x_{2}^{A-1}-1 & x_{2}^{B-1}-1
\end{array}\right| \neq 0 .
$$

Proof:

$$
\left|\begin{array}{ll}
x_{1}^{A-1}-1 & x_{1}^{B-1}-1 \\
x_{2}^{A-1}-1 & x_{2}^{B-1}-1
\end{array}\right|=0
$$

can be written as

$$
x_{1}^{A-1} x_{2}^{B-1}-x_{1}^{B-1} x_{2}^{A-1}-x_{1}^{A-1}+x_{1}^{B-1}-x_{2}^{B-1}+x_{2}^{A-1}=0 .
$$

Since

$$
x_{1}^{A-1}-1 \neq 0, x_{2}^{A-1}-1 \neq 0
$$

(3.17) can be written as

$$
\frac{x_{1}^{A-1}}{x_{1}^{A-1}-1}\left(x_{1}^{B-A}-1\right)=\frac{x_{2}^{A-1}}{x_{2}^{A-1}-1}\left(x_{2}^{B-A}-1\right) .
$$

When we define

$$
f(x)=\frac{x^{B}-x^{A}}{x^{A}-1}
$$

$f\left(x_{1}\right)=f\left(x_{2}\right)$ holds. Since

$$
f^{\prime}(x)=\frac{x^{A-1}}{\left(x^{A}-1\right)^{2}}\left((B-A) x^{B}-B x^{B-A}+A\right),
$$

define

$$
g(x)=\left((B-A) x^{B}-B x^{B-A}+A\right) .
$$

Note

$$
g^{\prime}(x)=(B-A) B x^{B-A-1}\left(x^{A}-1\right) .
$$

Therefore, on $0<x<1, g(x)$ is monotonely decreasing, and $g(1)=0$ holds, which implies that on $0<x<1, g(x)>0$. Namely, if $0<x<1$ and $1<A<B$,

$$
(B-A) x^{B}-B x^{B-A}+A>0
$$

stands. Therefore, since $f^{\prime}(x)>0$ on $0<x<1, f$ is monotonely increasing. Consequently, for $0<x_{1}, x_{2}<1, f\left(x_{1}\right)=f\left(x_{2}\right)$ implies $x_{1}=x_{2}$. By Lemma 3.8, for the case in which only one pair in $\left\{l_{l l}, l_{12}, l_{21}, l_{22}\right\}$ is duplicated, (3.13) cannot be satisfied.

Therefore, to satisfy (3.13),

$$
\left(l_{11}, l_{12}\right)=\left(l_{21}, l_{22}\right)
$$

should be satisfied. By substituting the value of $\left\|x_{i}-x_{j}\right\|$ in $(3.14), \sigma_{11}=\sigma_{21}$ and $\sigma_{12}=\sigma_{22}$ is obtained. This concludes the proof of Theorem 2.2. 


\section{Numerical Examples}

\subsection{RBF-Periodic mixed kernel GPR model}

\subsection{1. no $x \in X$ which satisfies $\frac{x}{p} \notin \mathbb{N}$}

In this example, we set $p=7$. Suppose the set of observed covariates is $\left\{x_{i}: 1 \leq i \leq n\right\}=$ $\{1,8,15, \ldots ., 7 n-6\}, n \geq 3$. In this case, if $\sigma_{1}=\sigma_{2}, l_{1}=l_{2}, \tau_{1}=\tau_{2}$ and $s_{1} \neq s_{2}$, then

$$
G_{R B F}\left(\theta_{1}\right)+G_{\text {period }}\left(\eta_{1}\right)=G_{R B F}\left(\theta_{2}\right)+G_{\text {period }}\left(\eta_{2}\right)
$$

is always satisfied. As as specific example, consider

$$
\left\{x_{i}: 1 \leq i \leq 6\right\}=\{1,8,15,22,29,36\} .
$$

If the mixed kernel is

$$
\sigma_{1}=\sigma_{2}=l_{1}=l_{2}=\tau_{1}=\tau_{2}=1, s_{1}=1 \text { and } s_{2}=2,
$$

then

$$
G=G_{R B F}\left(\theta_{1}\right)+G_{\text {period }}\left(\eta_{1}\right)=G_{R B F}\left(\theta_{2}\right)+G_{\text {period }}\left(\eta_{2}\right)=\left(\begin{array}{cccccc}
2 & 1 & 1 & 1 & 1 & 1 \\
1 & 2 & 1 & 1 & 1 & 1 \\
1 & 1 & 2 & 1 & 1 & 1 \\
1 & 1 & 1 & 2 & 1 & 1 \\
1 & 1 & 1 & 1 & 2 & 1 \\
1 & 1 & 1 & 1 & 1 & 2
\end{array}\right)
$$

This is a non-identifiable case where $X$ does not contain any element $x$ which satisfies $\frac{x}{p} \notin \mathbb{N}$.

4.1.2. no $x \in X$ which satisfies $\frac{x}{p} \in \mathbb{N}$

We consider the case that $\left\{x_{i}: 1 \leq i \leq n\right\}$ doesn't contain any element $x$ such that $\frac{x}{p} \in \mathbb{N}$. Suppose that $p=4$. When $X=\{0,1,2,3\}$, X doesn't contain any element $\mathrm{X}$ which satisfies $\frac{x}{p} \in \mathbb{N}$. For

$$
\begin{aligned}
& \sigma_{1}=1.5720871, l_{1}=1.0045602, \tau_{1}=1.4284245, s_{1}=1.2011224 \\
& \sigma_{2}=1.2295748, l_{2}=1.4468554, \tau_{2}=1.7320508, s_{2}=0.9540646
\end{aligned}
$$

$G_{R B F}\left(\theta_{1}\right)+G_{\text {period }}\left(\eta_{1}\right)=G_{R B F}\left(\theta_{2}\right)+G_{\text {period }}\left(\eta_{2}\right)$ holds. Define

$$
v(x)=\left(\begin{array}{c}
\exp \left(-\frac{x^{2}}{l_{1}^{2}}\right) \\
\exp \left(-\frac{x^{2}}{l_{2}^{2}}\right) \\
\exp \left(-\frac{\cos (2 \pi x / p)}{s_{1}^{2}}\right) \\
\exp \left(-\frac{\cos (2 \pi x / p)}{s_{2}^{2}}\right)
\end{array}\right) .
$$


The parameters above are obtained by solving the relation below analytically:

$$
v(0)-3 v(1)+6 v(2)-2 v(3)=(0,0,0,0) .
$$

Note in this case,

$$
\begin{aligned}
G= & G_{R B F}\left(\theta_{1}\right)+G_{\text {period }}\left(\eta_{1}\right)=G_{R B F}\left(\theta_{2}\right)+G_{\text {period }}\left(\eta_{2}\right)= \\
& \left(\begin{array}{llll}
4.5118543 & 1.9376702 & 0.5570357 & 1.0205292 \\
1.9376702 & 4.5118543 & 1.9376702 & 0.5570357 \\
0.5570357 & 1.9376702 & 4.5118543 & 1.9376702 \\
1.0205292 & 0.5570357 & 1.9376702 & 4.5118543
\end{array}\right) .
\end{aligned}
$$

\subsection{2-RBF mixed kernel GPR model}

For 2-RBF mixed kernel GPR model in 3-dimensional space, observations on 6 different points can even be insufficient for the identifiability. For example, assign 6 points coordinate as $( \pm 1, \pm 1,0),(0,0, \pm \sqrt{2})$, and parameters as follows:

$$
\begin{aligned}
& \sigma_{11}=24, \sigma_{12}=32 \sqrt{15}, l_{11}=\frac{1}{\sqrt{\log 4}}, l_{12}=\frac{1}{\sqrt{\log 16}} \\
& \sigma_{21}=81, \sigma_{22}=25 \sqrt{15}, l_{11}=\frac{1}{\sqrt{\log 9}}, l_{12}=\frac{1}{\sqrt{\log 25}}
\end{aligned}
$$

Then, $6 \times 6$ matrix $G_{R B F}\left(\theta_{11}\right)+G_{R B F}\left(\theta_{12}\right)$ and $G_{R B F}\left(\theta_{21}\right)+G_{R B F}\left(\theta_{22}\right)$ are identical and

$$
\begin{aligned}
G= & G_{R B F}\left(\theta_{11}\right)+G_{R B F}\left(\theta_{12}\right)=G_{R B F}\left(\theta_{21}\right)+G_{R B F}\left(\theta_{22}\right)= \\
& \left(\begin{array}{ccccccc}
15936 & 1104 & 1104 & 96 & 1104 & 1104 \\
1104 & 15936 & 96 & 1104 & 1104 & 1104 \\
1104 & 96 & 15936 & 1104 & 1104 & 1104 \\
96 & 1104 & 1104 & 15936 & 1104 & 1104 \\
1104 & 1104 & 1104 & 1104 & 15936 & 96 \\
1104 & 1104 & 1104 & 1104 & 96 & 15936
\end{array}\right) .
\end{aligned}
$$

In terms of model interpretation, the model explained by $\theta_{11}$ and $\theta_{12}$ poses an emphasis on long term smoothness, and the model by $\theta_{21}$ and $\theta_{22}$ on the short term smoothness, relatively. This non-identifiable situation occurs because $|X|=3$.

\section{Discussion}

In this paper, we define the identifiability of mixed kernel GPR models. Then we prove the theorems regarding the identifiability of two simple mixed GPR models, which are RBF-Periodic mixed GPR model and 2-RBF mixed GPR model. After analytic proofs, we give some numerical examples about non-identifiable cases regarding the models. Nonidentifiability is derived from lack of satisfaction of corresponding conditions. 


\section{Acknowledgements}

Jaeyong Lee was supported by the National Research Foundation of Korea (NRF) grants funded by the Korean government (MSIT) (No. 2018R1A2A3074973 and 2020R1A4A1018207).

\section{References}

Atienza, N., Garcia-Heras, J., Munoz-Pichardo, J. (2006). A new condition for identifiability of finite mixture distributions, Metrika, 63, 215-221.

Barndorff-Nielsen, O. (1965). Identifiability of mixtures of exponential families, Journal of Mathematical Analysis and Applications 12, 115-121.

Bousquet, O., von Luxburg, U., Rätsch, G. (2011). Advanced lectures on machine learning: ML Summer Schools 2003, Canberra, Australia, February 2-14, 2003, Tübingen, Germany, August 4-16, 2003, Revised Lectures, 3176, Springer.

Choi, J., Lee, K. (2017). Poisson linear mixed models with arma random effects covariance matrix, Journal of the Korean Data and Information Science Society, 28, 927-936.

Daemi, A., Alipouri, Y., Huang, B. (2019). Identification of robust gaussian process regression with noisy input using em algorithm. Chemometrics and Intelligent Laboratory Systems, 191, 1-11.

Gelman, A., Carlin, J.B., Stern, H.S., Dunson, D.B., Vehtari, A., Rubin, D.B. (2013). Bayesian data analysis, CRC press.

Huang, M., Li, R., Wang, H., Yao, W. (2014). Estimating mixture of gaussian processes by kernel smoothing, Journal of Business \& Economic Statistics, 32, 259-270.

Kostantinos, N. (2000). Gaussian mixtures and their applications to signal processing. Advanced signal processing handbook: Theory and implementation for radar, sonar, and medical imaging real time systems, 1-35, CRC Press.

Li, Z., Guo, F., Chen, L., Hao, K., Huang, B. (2020). Hybrid kernel approach to gaussian process modeling with colored noises, Computers \& Chemical Engineering, 143, 107067.

Noh, M., Ok, Y.J., Na, M.H., Ng, C.T. (2018). Analysis of degradation data using double hierarchical generalized linear model, The Korean Data \& Information Science Society, 29, 217-228.

Teicher, H. (1963). Identifiability of finite mixtures. The annals of Mathematical statistics, 1265-1269.

Wilson, A., Adams, R. (2013). Gaussian process kernels for pattern discovery and extrapolation. In International conference on machine learning, 1067-1075, PMLR

Yakowitz, S.J., Spragins, J.D. (1968). On the identifiability of finite mixtures. The Annals of Mathematical Statistics, 209-214. 